\title{
Efectos de un programa de actividad física en la imagen corporal de escolares con tdah
}

\author{
Effects of a physical activity program on the body image \\ of schoolchildren with adhd
}

\section{Efeitos de um programa de actividade física na imagem corporal de escolares com tdah}

\author{
Laura López Sánchez, Guillermo Felipe López Sánchez y Arturo Díaz Suárez
}

Facultad de Ciencias del Deporte, Universidad de Murcia, España

Resumen: Este artículo se centra en estudiar los efectos de un programa de actividad física sobre la imagen corporal de un grupo de escolares con TDAH. Han participado 12 escolares (12 niños), entre los 7 y los 12 ańos de edad. La imagen corporal se ha medido mediante las siluetas de Stunkard. El procedimiento ha sido: pre-test, intervención y pos-test. La intervención ha consistido en 2 días a la semana de actividad física, 60 minutos al día, durante 12 semanas. Se han producido mejoras significativas en la imagen corporal $(p=0,039)$. El programa utilizado es eficaz para mejorar la imagen corporal de nińos con TDAH.

Palabras clave: Imagen Corporal, Actividad Física, Educación Física, Educación Primaria, TDAH.

Abstract: This paper focuses on studying the effects of a physical activity program on body image of a group of schoolchildren with ADHD. This investigation involved 12 students (12 boys), aged between 7 and 12 years. The body image was measured by Stunkard's silhouettes. The procedure was as follows: pre-test, intervention and post-test. The intervention consisted of 2 days per week of physical activity, 60 minutes per day, during 12 weeks. There have been significant improvements in body image $(\mathrm{p}=0,039)$. The program used is effective to improve the body image of children with ADHD.

Keywords: Body Image, Physical Activity, Physical Education, Elementary Education, ADHD.

Resumem: Este artigo centra-se em estudar os efeitos de um programa de actividade física sobre a imagem corporal de um grupo de escolares com TDAH. Têm participado 12 escolares (12 meninos), entre os 7 e os 12 anos de idade. A imagem corporal mediu-se mediante as silhuetas de Stunkard. $\mathrm{O}$ procedimento tem sido: pré-teste, intervençăo e pos-teste. A intervenção tem consistido em 2 dias à semana de actividade física, 60 minutos ao dia, durante 12 semanas. Produziram-se melhoras significativas na imagem corporal $(\mathrm{p}=0,039)$. O programa utilizado é eficaz para melhorar a imagem corporal de meninos com TDAH.

Palavras-chave: Imagem Corporal, Actividade Física, Educação Física, Educação Primária, TDAH.

\section{Introducción}

En 1993, Michanie et al. afirmaban que aún no se disponía de un tratamiento curativo para este trastorno, pero ya proponían una serie de medidas terapéuticas eficaces para reducir las manifestaciones, consistentes en la combinación de un abordaje farmacológico (mediante estimulantes) y distintas intervenciones psicoterapéuticas (técnicas de orientación y entrenamiento a padres, de tipo cognitivo-conductual, con el objetivo disminuir el estrés que estas familias suelen presentar).

En las últimas décadas ha aumentado el interés por encontrar tratamientos que sean eficaces para reducir el TDAH (Barkley, 1997) y se han realizado diversas aportaciones. Existen tratamientos, en los que se incluyen el psicológico, el psiquiátrico, el farmacológico y la modificación de conducta (García, 2006). Asimismo, García y Hernández (2009) dis-

Dirección para correspondencia [Correspondence address]: Guillermo F. López Sánchez. E-mail: gfls@um.es tinguen dos tipos de intervención: tratamiento farmacológico e intervención conductual, mientras que Sances (2009) habla de tres modalidades de intervención del TDAH: farmacológica, psicosocial y combinada (terapia farmacológica y psicosocial). Según Grau (2007), los niños TDAH están recibiendo tratamiento en un $\mathbf{9 7 . 4 \%}$ de los casos, de los cuales en un $64 \%$ de los casos reciben tratamiento combinado (tanto psicológico como farmacológico) seguido del tratamiento sólo farmacológico en un $20.2 \%$ de los casos y sólo psicológico en un $13.2 \%$. Un dato a destacar por tanto es que el $84.2 \%$ de los nińos con TDAH recibe medicación.

Félix (2006) señala la conveniencia de combinar, junto con la intervención psicopedagógica, el tratamiento farmacológico. En la misma línea, Bitaubé et al. (2009) indica que el tratamiento debe incorporar medidas farmacológicas y psicosociales. Fernández y Calleja (2004) defienden que el tratamiento más efectivo en el TDAH es el psicoestimulan- 
te. Según Ureña (2007), el tratamiento que ha demostrado mayor efectividad es el "multidisciplinar" que combina los siguientes tratamientos: Tratamiento psicológico, tratamiento farmacológico y tratamiento psicopedagógico. Igualmente, Herranz y Argumosa (2000) precisan que se debe establecer un tratamiento multidisciplinario del niño: Apoyo psicopedagógico, información exhaustiva y tratamiento farmacológico. También Rubió et al. (2006) y Jarque (2012) indicaron que el tratamiento en muchos casos ha de ser un tratamiento multimodal y contextualizado. Siguiendo a Millán (2009), esta intervención multimodal debe incluir psicoeducación y entrenamiento de padres, intervenciones psicológicas-conductuales o cognitivo-conductuales, intervenciones escolares y psicopedagógicas y si fuese necesario tratamiento farmacológico. Para Lora (2006), los pilares del tratamiento son: plan de acción, educación, tratamiento farmacológico, no farmacológico y revisiones periódicas.

Con respecto al tratamiento farmacológico, el tratamiento de elección es el metilfenidato por su eficacia, seguridad y coste-efectividad (Lora, 2006; García et al., 2008). En cuanto al tratamiento farmacológico diferente al metilfenidato, el fármaco no estimulante que más datos científicos tiene apoyando su eficacia y seguridad en niños y adolescentes con TDAH es la atomoxetina, y es el único indicado como de primera elección, junto con los estimulantes (Díez et al., 2006).

Respecto a los tratamientos no farmacológicos, en los últimos tiempos han aparecido nuevas líneas de interés, más allá de los habituales tratamientos conductuales o cognitivoconductuales (Cardo y Servera, 2008). Entre ellas, Cidoncha (2010) destaca el papel favorable de la Educación Física, debido a que permite trabajar la inhibición muscular, el control postural, la relajación y la autoestima, tan fundamental para ellos, ya que les beneficia en su rendimiento académico, sus relaciones sociales y su autoconocimiento. Rosal (2008) también propone una serie de actividades para tratar de facilitar en el niño/a con TDAH la relajación, el autocontrol, la atención, la concentración y la reducción de la tensión, entre las que incluye ejercicios de saltos, levantamientos de pesos y baile. Además se pueden proponer situaciones de resolución de problemas como estrategia de trabajo con nińos diagnosticados con TDAH (Ochoa et al., 2006). También son interesantes las intervenciones no farmacológicas del TDAH en el hogar o entorno familiar, las cuales incluyen informar a los padres sobre los diferentes aspectos del trastorno y de cómo pueden afectar a su hijo, e instruirles en nociones de terapia del comportamiento y terapia cognitiva para un mejor control de la conducta perturbadora, de la falta de organización y atención del niño (Eddy, 2006).

Una detección y tratamiento precoces ayudarán a controlar los síntomas, mejorando el aprendizaje escolar y las interacciones sociales del TDAH (García et al., 2008; Martínez de Haro et al., 2003). Destaca la situación actual, en la cual las familias, los profesores y orientadores psicopedagógicos, desconocedores de la naturaleza de este problema, de sus características y de las alternativas de tratamiento, se sienten incapaces de ofrecer ayuda adecuada a estos niños e incluso les malinterpretan en su conducta, procediendo a una cierta marginación y a la consideración de niños malos, revoltosos, rebeldes, etc. (San Sebastián, 2005). Es por ello de suma importancia desarrollar estrategias de formación (Guerrero y Pérez, 2011; Herranz, 2006) y que haya una adecuada cooperación entre el pediatra y el maestro, entre la escuela y el centro de salud (Rodríguez-Salinas et al., 2006). Además se deben tener en cuenta una serie de orientaciones educativas para niños con TDAH, como las propuestas por Cortés (2010), entre las que se pueden destacar la coordinación profesoresequipo de orientación-familias, estrategias de afrontamiento y resolución de problemas, disciplina y buena relación afectiva, dar la oportunidad de desarrollar lo que saben hacer bien, no realizar tareas largas y complejas, técnicas de modificación de conducta: elogiar, recompensar y establecer límites.

Es necesaria la realización de más investigaciones sobre estas cuestiones para poder precisar mejor sus implicaciones y elaborar adecuadas estrategias para la prevención y el tratamiento del TDAH y de sus complicaciones. Nuestra propuesta plantea, por tanto, un tratamiento no farmacológico innovador, basado en la realización de actividad física de forma regular (dos días a la semana), el cual tiene como objetivo mejorar la salud de los nińos y adolescentes con TDAH, prestando especial atención a la mejora de la imagen corporal de los escolares con TDAH.

Esta propuesta se apoya en otros trabajos anteriores, como el de Garza-Morales et al. (2007) que mostraron que los niños con TDAH presentan menores niveles de autoestima y autoconcepto que los niños que no presentan el trastorno, o el de Frame et al. (2003) que encontraron mejoras en la apariencia física percibida de un grupo de escolares con TDAH tras un programa de intervención no farmacológico consistente en reuniones con un grupo de apoyo guiadas por enfermeras especializadas en el trastorno.

\section{Método}

\section{Características de los participantes y muestra}

La muestra del estudio se compone de 12 escolares de sexo masculino, con una media de edad de 9.83 años y un rango de edad de 7 a 12 ańos. A los 12 escolares les había sido diagnosticado TDAH (Trastorno por déficit de atención con hiperactividad) y estaban recibiendo tratamiento farmacológico.

Esta investigación ha sido aprobada por la Comisión de Ética de Investigación de la Universidad de Murcia y todos los participantes han firmado un consentimiento informado. 


\section{Diseño y variables de estudio}

El diseño ha sido un diseño cuasiexperimental pre-post con un solo grupo de intervención ya que el alumnado fue seleccionado por presentar el trastorno, de forma no aleatoria, y se consideró como aspecto primordial de la investigación que todos los alumnos con TDAH recibieran tratamiento.

Los sujetos participantes en el estudio han realizado un pre-test, una intervención de 12 semanas y un pos-test.

En el pre-test se ha analizado la variable Imagen Corporal.

Durante la intervención, los escolares participantes han realizado sesiones de actividad física aeróbica y de intensidad media-alta, dos días a la semana (60 minutos cada día) fuera del horario lectivo, en las que los alumnos han llevado a cabo circuitos y ejercicios destinados a mejorar su condición física, especialmente la inhibición muscular y el control postural, enfatizando en la relajación y en la autoestima. La autoestima se ha favorecido mediante diversas estrategias en las actividades realizadas: proponer logros alcanzables, graduar de menor a mayor dificultad, promover situaciones de éxito y utilizar recompensas para reforzar los logros conseguidos; además se han utilizado las estrategias propuestas por Mínguez \& Ortega (1999) para la mejora de la autoestima en el ámbito escolar: técnicas de aprendizaje cooperativo y de participación activa, promoviendo el descubrimiento y aceptación de "mi realidad como persona y orientación de mi vida hacia un proyecto valioso". Las sesiones han sido dirigidas por el personal investigador.

En el pos-test se ha vuelto a analizar la imagen corporal de los escolares para comprobar si se han producido mejoras respecto al pre-test.

\section{Procedimiento}

Tras la revisión de la literatura, se han mantenido diferentes reuniones entre el personal investigador para organizar la obtención de datos y revisar los protocolos de medición. Además se han realizado reuniones con los responsables de la Asociación de Ayuda al Déficit de Atención con más o menos Hiperactividad de Murcia (ADAHI) y con los padres de los sujetos de la muestra, para concretar las fechas y horarios del pretest, la intervención y el postest. Igualmente, se han llevado a cabo reuniones periódicas con el objetivo de realizar un seguimiento sistemático del trabajo realizado.

También se hizo entrega a los padres de un modelo de consentimiento informado en donde se detallan las pruebas y objetivos de la investigación. Tras el visto bueno paterno, los investigadores comenzaron con la recogida de datos y la intervención.

El enfoque metodológico en la intervención se ha llevado a cabo desde una perspectiva fundamentalmente lúdica. Contando con la motivación natural de los alumnos hacia el juego y la actividad deportiva y conduciéndola hacia la cooperación, el respeto a las reglas, el esfuerzo por superar las dificultades, la autonomía y la alegría por la tarea bien hecha.

\section{Instrumentos}

Se ha estudiado la imagen corporal porque en ocasiones el TDAH se asocia a bajos niveles de autoestima e imagen corporal (Frame et al., 2003) y el autoconcepto suele ser menor en los niños con TDAH (Garza-Morales et al., 2007). Las siluetas de Stunkard son una sencilla forma de medir la imagen corporal. Muestran nueve figuras de siluetas corporales, tanto de varones como de mujeres, que corresponden a diferentes índices de masa corporal. Los sujetos deben elegir la figura que más se aproxima a su silueta y también la silueta a la que les gustaría parecerse. Los resultados ofrecen tres medidas: la imagen actual, la imagen deseada y la discrepancia (deseada-actual), la cual es interpretada como una medida de insatisfacción con la imagen corporal. Si la discrepancia es igual a 0 , el sujeto está satisfecho con su imagen corporal; si la discrepancia tiene valor positivo, el sujeto tiene un deseo de ser más grande; si la discrepancia tiene un valor negativo, el sujeto tiene un deseo de ser más delgado. Las siluetas de Stunkard ofrecen una alta fiabilidad para clasificar sujetos obesos -0.93 para mujeres y 0.88 para hombres- o delgados -0.87 para mujeres y 0.88 para hombres- (Bulik et al., 2001).

Figura 1. Siluetas de Stunkard.

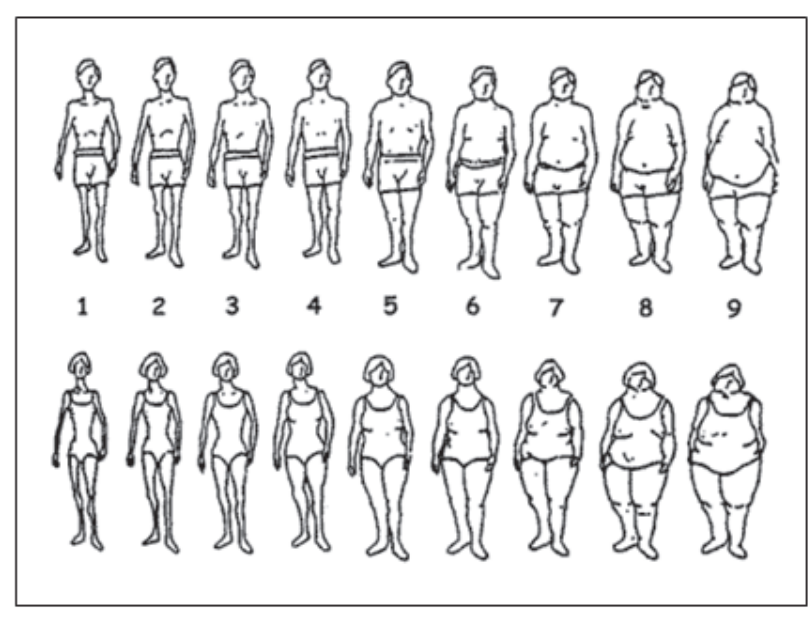

\section{Análisis de datos}

Se ha realizado un análisis estadístico por medio del Statistical Package for Social Sciences 22.0 (SPSS-22.0). Se han seguido las indicaciones del Manual de estadística aplicada a las Ciencias de la Actividad Física y el Deporte (Ortega, 
Ortiz y Artés, 2009) y se ha presentado lo que se indica a continuación:

- Estadísticos descriptivos de cada ítem o prueba: mínimo, máximo, media y desviación estándar.

- Análisis de frecuencias y porcentajes de cada ítem o prueba.

- Prueba de Wilcoxon para muestras relacionadas. Esta prueba permite saber si se han producido diferencias significativas entre el pre-test y el pos-test.

\section{Resultados}

A continuación se presentan los resultados en forma de tablas:

La tabla 1 es un resumen de los estadísticos descriptivos (mínimo, máximo, media y desviación estándar). En el pretest se observa una imagen corporal media de 0.33 , es decir, los sujetos tienen un deseo de ser más grandes. En cambio, en el postest, la imagen corporal media adquiere un valor negativo, que indica un deseo de ser más delgado.

Tabla 1. Estadísticos descriptivos.

\begin{tabular}{lccccc}
\hline $\begin{array}{l}\text { Imagen } \\
\text { corporal }\end{array}$ & N & Mínimo & Máximo & Media & $\begin{array}{c}\text { Desviación } \\
\text { estándar }\end{array}$ \\
\hline Pretest & 12 & $-2,00$ & 2,00 & 0,3333 & 1,49747 \\
Postest & 12 & $-3,00$ & 1,00 & $-0,9167$ & 1,31137 \\
\hline
\end{tabular}

En la tabla 2, se realiza un análisis en función de frecuencias y porcentajes. Es destacable que el número de escolares satisfechos se incrementa de 1 hasta 5 y que el número de escolares que deseaba ser más grande se reduce de 7 a 1 .

Tabla 2. Frecuencias y porcentajes.

\begin{tabular}{lcccccc}
\hline \multirow{2}{*}{$\begin{array}{l}\text { Imagen } \\
\text { corporal }\end{array}$} & \multicolumn{2}{c}{ Satisfecho } & \multicolumn{2}{c}{$\begin{array}{c}\text { Deseo de ser más } \\
\text { delgado }\end{array}$} & \multicolumn{2}{c}{$\begin{array}{c}\text { Deseo de ser más } \\
\text { grande }\end{array}$} \\
\cline { 2 - 7 } & Frec. & $\%$ & Frec. & $\%$ & Frec. & $\%$ \\
\hline Pretest & 1 & 8,33 & 4 & 33,33 & 7 & 58,33 \\
Postest & 5 & 41,7 & 6 & 50 & 1 & 8,3 \\
\hline
\end{tabular}

Por último, en la tabla 3 se analizan de forma más detallada las diferencias entre el pre-test y el pos-test de la muestra $(\mathrm{n}=12)$, mostrando los niveles de imagen corporal de los 12 sujetos que completaron pre-test, intervención y pos-test.
Tabla 3. Comparación pre-post muestra final $(n=12)$.

\begin{tabular}{rlrl}
\hline Imagen corporal pre-test $(\mathrm{n}=12)$ & Imagen corporal pos-test $(\mathrm{n}=12)$ \\
\hline-1 & Deseo más delgado & -3 & Deseo más delgado \\
1 & Deseo más grande & 0 & Satisfecho \\
-2 & Deseo más delgado & -2 & Deseo más delgado \\
2 & Deseo más grande & 0 & Satisfecho \\
0 & Satisfecho & 0 & Satisfecho \\
1 & Deseo más grande & 0 & Satisfecho \\
2 & Deseo más grande & 0 & Satisfecho \\
1 & Deseo más grande & -3 & Deseo más delgado \\
-1 & Deseo más delgado & -1 & Deseo más delgado \\
1 & Deseo más grande & 1 & Deseo más grande \\
2 & Deseo más grande & -1 & Deseo más delgado \\
-2 & Deseo más delgado & -2 & Deseo más delgado \\
\hline
\end{tabular}

Al aplicar la prueba de Wilcoxon para muestras relacionadas $(n=12)$, se observa que $\mathrm{p}=0.017$, por lo que se puede afirmar que, debido a que la significación obtenida es menor que 0.05 , se han producido diferencias significativas entre el pretest $y$ el postest.

\section{Discusión}

En cuanto al análisis descriptivo de los parámetros evaluados, se observa que, respecto a la imagen corporal, en el pretest solo el 8,33\% están satisfechos con la suya, lo que indica problemas con su imagen corporal, como se aprecia en la investigación de Garza-Morales et al. (2007), que evaluaron la autoestima y el autoconcepto de 60 niños mexicanos (30 con TDAH y 30 controles) de nivel socioeconómico alto y con edades comprendidas entre 6 y 13 años, de los cuales 40 fueron de género masculino y 20 de género femenino, siendo la proporción de varones/mujeres de 20/10 respectivamente para TDAH y controles. Los resultados mostraron valores de autoestima y autoconcepto significativamente mayores en el grupo control; los propios autores indican que estos valores significativamente más bajos en los niños con TDAH podrían representar una consecuencia de la problemática social y escolar, relacionada con su baja competitividad e inseguridad.

En cuanto a los efectos de la intervención, no hay muchas investigaciones que hayan evaluado los efectos de una intervención en la imagen corporal de escolares con TDAH. No obstante, nuestros resultados pueden ser comparados con el trabajo de Frame et al. (2003), que evaluaron la efectividad de un programa de intervención en la competencia deportiva percibida y la apariencia física percibida de un grupo de 65 preadolescentes estadounidenses con TDAH de clase mediaalta. El diseño que utilizaron fue cuasi-experimental, de tal 
forma que los sujetos fueron asignados de manera aleatoria al grupo control o al experimental. Todos los sujetos fueron evaluados mediante el "Perfil de Auto-Percepción de Harter", realizando un pretest y un postest 4 semanas después. En los sujetos del grupo experimental se llevó a cabo un programa de intervención de 4 semanas, en el que los alumnos se reunían dos veces a la semana con un grupo de apoyo de enfermeras especializadas. Los resultados mostraron mejoras significativas en la competencia deportiva percibida y la apariencia física percibida del grupo experimental, por lo que se puede concluir que la participación en un programa de intervención de este tipo está asociado positivamente con la mejora de la competencia deportiva percibida y la apariencia física percibida, en escolares con TDAH.

Como se puede observar, los resultados de esta investigación son similares a los de Frame et al. (2003), en la medida en que en ambos se encontraron mejoras significativas en la imagen corporal o autoconcepto de escolares con TDAH tras un programa de intervención no farmacológico.

Los resultados de esta investigación también pueden ser comparados con los de otras investigaciones, que aunque no hayan focalizado su atención en la imagen corporal de los escolares con TDAH, sí han llevado a cabo programas de intervención para intentar mitigar los síntomas del trastorno, consiguiendo resultados positivos. Se plantean diferentes tipos de intervenciones como la de Fernández et al. (2003), que llevan a cabo una intervención cognitivo-conductual basada en la formación de padres, docentes y alumnado, la cual consigue producir mejoras significativas en las conductas problema que presentaban los alumnos en el contexto escolar. Otra propuesta de intervención educativa es la de Escalera (2009), que interviene con el alumno, con sus compañeros y con sus padres, mediante un tratamiento cognitivo-conductual (técnica de ganancia de puntos, uso de reforzadores, actividades de relajación muscular y actividades de valores y conductas). O el programa de intervención multidisciplinar que plantea Ruiz (2010), consistente en la formación de tutores, familiares y profesorado y en la realización de actividades encaminadas a la potenciación de la atención, las habilidades sociales y el autocontrol de los alumnos. Es también destacable la intervención llevada a cabo por Presentación et al. (2010) con niños con TDAH, sus padres y sus profesores, la cual incluyó modificación de conducta, técnicas cognitivo-conductuales, adaptaciones académicas y habilidades sociales y consiguió mejoras duraderas tras el tratamiento, especialmente en las áreas académica y social.

Entre las intervenciones, ocupan un papel destacado las intervenciones mediante actividad física. Gapin et al. (2011) revisan la evidencia existente sobre los efectos de la actividad física en los síntomas del TDAH y señalan que la actividad física puede beneficiar los síntomas de comportamiento y el rendimiento cognitivo de los niños con TDAH, por lo que podría ser un complemento eficaz a la medicación o un tratamiento alternativo para aquellos niños que no responden a los tratamientos de medicación o desean buscar formas alternativas de tratamiento. Esta es también la hipótesis de Wigal et al. (2012) que plantean que el ejercicio físico altera la fisiología subyacente presente en el TDAH y podría constituir una importante alternativa y/o un tratamiento complementario al farmacológico. En esta línea, Mena et al. (2008), aplicaron una estrategia pedagógica basada en la psicomotricidad a través de muchas actividades como el baile y el juego y encontraron que los nińos con TDAH trabajaban mejor y disfrutaban más de las actividades académicas en la escuela, a la vez que reducían sus niveles de hiperactividad y de déficit de atención. Otro estudio interesante es el de Azrin et al. (2007), sobre el uso de la actividad física como refuerzo para la tranquilidad de los niños con TDAH en el aula, que mostró un aumento de la calma en los niños durante la duración de la clase. O el de Barnard-Brak et al. (2011), que establecieron una asociación entre la Educación Física y los síntomas del TDAH, sugiriendo que la Educación Física, como una forma estructurada de la actividad física, se puede considerar asociada con menores niveles de los síntomas del TDAH a lo largo del tiempo.

Otro tipo de intervención mediante actividad física que ha mostrado resultados positivos es la de los campamentos de verano. Así, Gerber-von Muller et al. (2009), en un campamento de verano intensivo con niños y adolescentes con TDAH, desarrollaron y evaluaron un programa de intervención multimodal (entrenamiento de habilidades sociales, actividades deportivas y medicación), consiguiendo mejoras significativas de larga duración en los síntomas del TDAH tras el campamento de verano. Igualmente, Gerber et al. (2012) aplicaron un tratamiento multimodal (metilfenidato, entrenamiento de habilidades sociales, entrenamiento de la atención y participación en deportes), en el formato de un campamento de verano intensivo, y obtuvieron mejoras duraderas en las funciones neuropsicológicas de los niños y adolescentes con TDAH. De forma similar, Yamashita et al. (2011) llevaron a cabo un programa multidisciplinar de tratamiento de verano, en el cual se incluyó entrenamiento en habilidades deportivas, sociales y académicas, en nińos con TDAH y los resultados mostraron que la mayoría de niños consiguió cambios positivos en el comportamiento y en algunas funciones cognitivas. Por último, Hupp et al. (2002), mediante un programa de verano, mostraron que el uso de recompensas y alabanzas puede mejorar la conducta deportiva de los niños con TDAH.

Son también relevantes otras investigaciones como la de Tantillo et al. (2001) que evaluaron los efectos del ejercicio en nińos con TDAH, obteniendo resultados positivos que alientan a realizar nuevos estudios con el fin de corroborar si una sesión de ejercicio vigoroso tiene eficacia en la gestión del 
comportamiento del TDAH. O la de Kang et al. (2011), que llevaron a cabo una terapia mediante deporte en nińos con TDAH, cuyos resultados demostraron una correlación positiva entre el deporte y la mejora de los síntomas de la atención, los síntomas cognitivos y las habilidades sociales, por lo que los autores del estudio sugirieron que la terapia mediante deporte puede aliviar los síntomas de atención y aumentar la competencia social en los niños con TDAH. En la misma línea, Smith et al. (2013) llevaron a cabo una intervención mediante actividad física en niños con TDAH y los resultados mostraron que la mayoría de los participantes mostraron mejoría general después del programa, por lo que los autores sugieren que la actividad física se muestra prometedora para tratar los síntomas del TDAH. Kiluk et al. (2009) también sugirieron que la participación activa en deportes puede estar asociada a menores niveles de ansiedad o depresión en niños con TDAH. Asimismo, en el programa terapéutico basado en la práctica deportiva de Lufi y Parish-Plass (2011), llevado a cabo durante un año académico, se produjeron mejoras en el comportamiento y se redujo la ansiedad de los niños con TDAH.

Otros autores que han contribuido a la investigación en este campo son Patel y Curtis (2007), los cuales realizaron un tratamiento multidimensional (nutrición, control del ambiente y terapia comportamental, educacional, física y del lenguaje) en niños con TDAH y documentaron que todos los niños mostraron mejoras significativas en las áreas de interacción social, concentración, escritura, lenguaje y comportamiento. Igualmente, Pontifex et al. (2013) mostraron que el ejercicio mejora el comportamiento, la atención y la actuación académica en niños con TDAH, de tal forma que sesiones individuales de ejercicio aeróbico de intensidad moderada pueden tener implicaciones positivas en la función neurocognitiva y el control inhibitorio en niños con TDAH. Además parece ser que las actividades al aire libre reducen los síntomas del TDAH (Kuo y Faber, 2004), como la equitación (Rubio y García, 2011). Por último, cabe destacar el estudio de Medina et al. (2010), midieron el impacto de actividad física de alta intensidad en la atención sostenida niños diagnosticados con TDAH y encontraron que los déficits de atención de los niños pueden ser minimizados a través de actividad física, aunque se necesitan más estudios que confirmen que el ejercicio mitiga los síntomas del TDAH.

Por tanto, tras comparar este estudio con otros trabajos de investigación relacionados, se puede afirmar que este trabajo presenta una intervención mediante actividad física, pero realiza una innovadora aportación. La intervención no se centra sólo en mitigar los principales síntomas del TDAH (inatención y/o hiperactividad-impulsividad), sino que también intenta mejorar otra área donde los niños con TDAH suelen presentar problemas y que ha sido menos estudiada hasta el momento. Esta área es la imagen corporal, y según los resultados de este trabajo, puede ser mejorada en los escolares con TDAH a través de un programa de intervención mediante actividad física.

\section{Aplicaciones prácticas}

El TDAH puede presentarse asociado a problemas en la variable imagen corporal. Una intervención mediante actividad física de intensidad media-alta, con dos sesiones por semana de 60 minutos cada una, puede producir mejoras significativas en la imagen corporal de niños con TDAH.

Se recomienda llevar a cabo otros programas de intervención mediante actividad física, de diferente duración y con diferentes metodologías, y medir los efectos de los mismos sobre la salud de los niños con TDAH, de tal forma que haya más recursos disponibles para reducir los síntomas de este trastorno y mejorar los diferentes parámetros saludables de los niños con TDAH.

Financiación: Fundación Séneca - Agencia de Ciencia y Tecnología de la Región de Murcia. Consejería de Industria, Turismo, Empresa e Innovación de la CARM.

\section{Referencias bibliográficas}

1. Azrin, N. H., Vinas, V. y Ehle, C. T. (2007). Physical activity as reinforcement for classroom calmness of ADHD children: A preliminary study. Child y Family Behavior Therapy, 29 (2), 1-8.

2. Barkley, R. (1997). ADHD and the nature of self-control. New York: Guilford Press.

3. Barnard-Brak, L., Davis, T., Sulak, T. y Brak, V. (2011). The Association Between Physical Education and Symptoms of Attention Deficit Hyperactivity Disorder. Journal of Physical Activity y Health, 8 (7), 964-970.

4. Bitaubé, J. A., López-Martín, S., Fernández-Jaén, A. y Carretié Arangüena, L. (2009). Trastorno por déficit de atención con o sin hiperactividad. Jano, 1729, 18-26.

5. Bulik, C. M., Wade, T. D., Heath, A. C., Martin, N. G., Stunkard, A. J. y Eaves, L. J. (2001). Relating body mass index to figural stimuli: population-based normative data for Caucasians. International Journal of Obesity, 25, 1517-1524.
6. Cardo, E. y Servera, M. (2008). Trastorno por déficit de atención/hiperactividad: Estado de la cuestión y futuras líneas de investigación. Revista de Neurología, 46 (6), 365-372.

7. Cidoncha Delgado, A. I. (2010). Niños con Déficit de Atención por Hiperactividad TDAH: Una Realidad Social en el Aula. Revista Autodidacta, 1 (4), 31-36.

8. Cortés Ariza, M. C. (2010). Déficit de Atención con Hiperactividad (TDAH): Concepto, Características e Intervención Educativa. Innovación y Experiencias Educativas, 28, 1-8.

9. Díez Suárez, A., Figueroa Quintana, A. y Soutullo Esperón, C. (2006). Trastorno por déficit de atención e hiperactividad (TDAH): comorbilidad psiquiátrica y tratamiento farmacológico alternativo al metilfenidato. Revista Pediatría de Atención Primaria, 8 (4), $135-155$.

10. Eddy Ives, L. (2006). Intervenciones no farmacológicas en el entorno 
familiar de niños con trastorno por déficit de atención con/sin hiperactividad. Revista Pediatría de Atención Primaria, 8 (4), 57-67.

11. Escalera, M. R. (2009). Una rentable propuesta de intervención educativa para el niño con TDAH. Innovación y Experiencias Educativas, 16, 1-10.

12. Félix Mateo, V. (2006). Recursos para el diagnóstico psicopedagógico del TDAH y comorbilidades. Revista Electrónica de Investigación Psicoeducativa, 10, 4 (3), 623-642.

13. Fernández Martín, F., Hinojo Lucena, F. J. y Aznar Díaz, I. (2003). Dificultades del Alumnado con Trastorno por Déficit de Atención con Hiperactividad (TDAH) en el Aula: Implicaciones para la Formación Docente. Enseñanza, 21, 219-232.

14. Frame, K., Kelly, L. y Bayley, E. (2003). Increasing perceptions of selfworth in preadolescents diagnosed with ADHD. Journal of Nursing Scholarship, 35, 3, 225-229.

15. Gapin, J. I., Labban, J. D. y Etnier, J. L. (2011). The effects of physical activity on attention deficit hyperactivity disorder symptoms: The evidence. Preventive Medicine, 52 (1), S70-S74.

16. García Chávez, M. I. y Hernández Vicente, E. I. (2009). Modificación de conceptos a padres y profesores en relación al TDAH. Tesis Doctoral. Universidad Michoacana de San Nicolás de Hidalgo.

17. García García, M. D., Prieto Tato, L. M., Santos Borbujo, J., Monzón Corral, L., Hernández Fabián, A. y San Feliciano Martín, L. (2008). Trastorno por déficit de atención e hiperactividad: un problema actual. Anales de Pediatría, 69 (3), 244-250.

18. Garza-Morales, S., Núńez-Villaseñor, P. S. y Vladimirsky-Guiloff, A. (2007). Autoestima y locus de control en niños con trastorno por déficit de atención con hiperactividad. Mediagraphic, 64, 231-239.

19. Gerber-von Mueller, G., Petermann, U., Petermann, F., Niederberger, U., Stephani, U., Siniatchkin, M. y Gerber, W. D. (2009). ADHD summer camp: Development and evaluation of a multimodal intervention program. Kindheit und Entwicklung, 18 (3), 162-172.

20. Gerber, W. D., Gerber-von Mueller, G., Andrasik, F., Niederberger, U., Siniatchkin, M., Kowalski, J. T., Petermann, U. y Petermann, F. (2012). The impact of a multimodal Summer Camp Training on neuropsychological functioning in children and adolescents with ADHD: An exploratory study. Child Neuropsychology, 18 (3), 242-255.

21. Grau Sevilla, M. D. (2007). Análisis del Contexto Familiar en Niños con TDAH. Tesis Doctoral. Universidad de Valencia.

22. Guerrero López, J. F. y Pérez Galán, R. (2011). El alumnado con TDAH (hiperactividad) como colectivo en riesgo de exclusión social: propuestas de acción y de mejora. Revista RUEDES, 2, 37-59.

23. Herranz, J. L. y Argumosa, A. (2000). Neuropediatría. Trastorno con déficit de atención e hiperactividad. Boletín de la Sociedad de Pediatría de Asturias, Cantabria, Castilla y León, 40 (172), 88-92.

24. Herranz Jordán, B. (2006). Trastorno por déficit de atención e hiperactividad: conocimientos y forma de proceder de los pediatras de Atención Primaria. Revista Pediatría de Atención Primaria, 8 (4), 217-239.

25. Hupp, S., Reitman, D., Northup, J., O'Callaghan, P y LeBlanc, M. (2002). The Effects of Delayed Rewards, Tokens, and Stimulant Medication on Sportsmanlike Behavior With ADHD-Diagnosed Children. Behavior Modification, 26 (2), 148-162.

26. Jarque Fernández, S. (2012). Eficacia de las intervenciones con niños y adolescentes con Trastorno por Déficit de Atención con Hiperactividad (TDAH). Anuario de Psicología, 42 (1), 19-33.

27. Kang, K. D., Choi, J. W., Kang, S. G. y Han, D. H. (2011). Sports Therapy for Attention, Cognitions and Sociality. International Journal of Sports Medicine; 32, 953-959.

28. Kiluk, B. D., Weden, S. y Culotta, V. P. (2009). Sport Participation and Anxiety in Children With ADHD. Journal of Attention Disorders, 12 (6), 499-506.

29. Konikowska, K., Regulska-Ilow, B. y Rozanska, D. (2012). The influence of components of diet on the symptoms of ADHD in children. Roczniki Panstwowego Zakladu Higieny, 63, 2, 127-134.
30. Kuo, F. E., y Faber Taylor, A. (2004). A Potential Natural Treatment for Attention-Deficit/Hyperactivity Disorder: Evidence From a National Study. American Journal of Public Health, 94 (9), 1580-1586.

31. Lora Espinosa, A. (2006). El tratamiento del niño y adolescente con TDAH en Atención Primaria desde el punto de vista de la evidencia. Revista Pediatría de Atención Primaria, 8 (4), 69-114.

32. Lufi, D. y Parish-Plass, J. (2011). Sport-Based Group Therapy Program for Boys with ADHD or with Other Behavioral Disorders. Child y Family Behavior Therapy, 33 (3), 217-230.

33. Martínez de Haro, V., Álvarez Barrios, M. J., Cid Yagüe, L., Garoz Puerta, I., Vega Marcos, R. de la y Villagra Astudillo, A. (2003). Evaluación de la Salud en Educación Física. Educación Física y deporte escolar. Actas del $V$ Congreso Internacional de FEADEF. Ed. AVAPEF. Valladolid, 313-317.

34. Medina, J. A., Netto, T. L., Muszkat, M., Medina, A. C., Botter, D., Orbetelli, R., Scaramuzza, L. F., Sinnes, E. G., Vilela, M. y Miranda, M. C. (2010). Exercise impact on sustained attention of ADHD children, methylphenidate effects. Attention deficit and hyperactivity disorders, 2 (1), 49-58.

35. Mena Rodríguez, F. E., Salgado Muñoz, A. P. y Tamayo Marín, P. A. (2008). Estrategia Pedagógica basada en la Lúdica y Psicomotricidad aplicada en las Âreas de Castellano y ciencias Naturales para Centrar la Atención de Niños y Niñas con TDAH de Preescolar y Básica Primaria de la Institución Educativa Ciudadela Cuba. Tesis de Licenciatura. Universidad Tecnológica de Pereira.

36. Michanie, C., Márquez, M., Estévez, P. y Steimberg, L. (1993). Artículo especial. Trastorno por déficit de atención con hiperactividad (TDAH). Archivos Argentinos de Pediatría, 91, 1-12.

37. Millán Lara, L. (2009). El Alumno Adolescente con TDAH (Manual de Estrategias para Profesores de Educación Secundaria). Tesis de Licenciatura. Universidad Pedagógica Nacional. México, DF.

38. Mínguez Vallejos, R. \& Ortega Ruiz, P. (1999). La educación de la autoestima. Revista de educación, 320, 335-352.

39. Ochoa Angrino, S., Cruz Panesso, I. y Iván Valencia, A. (2006). Las situaciones de resolución de problemas como estrategia de trabajo con niños diagnosticados con TDAH. Pensamiento Psicológico, 2 (7), 73-88.

40. Ortega, E., Ortiz, I. M. y Artés, E. M. (2009). Manual de Estadística Aplicada a las Ciencias de la Actividad Física y el Deporte. Murcia: Diego Marín.

41. Patel, K. y Curtis, L. T. (2007). A comprehensive approach to treating autism and attention-deficit hyperactivity disorder: A prepilot study. Journal of Alternative and Complementary Medicine, 13 (10), 1091-1097.

42. Pontifex, M. B., Saliba, B. J., Raine, L. B., Picchietti, D. L. y Hillman, C. H. (2013). Exercise Improves Behavioral, Neurocognitive, and Scholastic Performance in Children with Attention-Deficit/Hyperactivity Disorder. The Journal of Pediatrics, 162 (3), 543-551.

43. Presentación, M. J., Siegenthaler, R., Jara, P. y Miranda, A. (2010). Seguimiento de los efectos de una intervención psicosocial sobre la adaptación académica, emocional y social de niños con TDAH. Psicothema, 22 (4), 778-783.

44. Rodríguez-Salinas Pérez, E., Navas García, M., González Rodríguez, P., Fominaya Gutiérrez, S. y Duelo Marcos, M. (2006). La escuela y el trastorno por déficit de atención con/sin hiperactividad (TDAH). Revista Pediatría de Atención Primaria, 8 (4), 175-198.

45. Rosal, I. M. (2008). Atención Educativa para el Alumnado con Trastorno por Déficit de Atención con Hiperactividad. Innovación y Experiencias Educativas, 9, 1-9.

46. Rubió Badía, I., Mena Pujol, B. y Murillo Abril, B. (2006). El pediatra y la familia de un niño con TDAH. Revista Pediatría de Atención Primaria, 8 (4), 199-216.

47. Rubio Jiménez, J. C. y García Gómez, A. (2011). Programa de iniciación a la equitación para alumnos con TEA y con TDAH. Consejería de Educación: Junta de Extremadura. 
48. Ruiz Triviño, L. (2010). Programa para Alumnado con TDAH. Innovación y Experiencias Educativas, 27, 1-15.

49. San Sebastián, J. (2005). Trastorno por Déficit de Atención con Hiperactividad. Un trastorno del comportamiento precursor de otros. I Congreso Internacional de Trastornos del Comportamiento.

50. Sances Masero, C. (2009). Intervención Familiar Grupal en Niños con Trastorno por Déficit de Atención con Hiperactividad (TDAH). Tesis de Licenciatura. Universidad Abat Oliba CEU.

51. Smith, A. L., Hoza, B, Linnea, K., McQuade, J. D., Tomb, M., Vaughn, A. J., Shoulberg, E. K. y Hook, H. (2013). Pilot Physical Activity Intervention Reduces Severity of ADHD Symptoms in Young Children. Journal of Attention Disorders, 17 (1), 70-82.

52. Ureña Morales, E. (2007). Guía Práctica con Recomendaciones de Ac- tuación para los Centros Educativos en los Casos de Alumnos con TDAH. Islas Baleares: STILL. Asociación Balear de Padres de Niños con TDAH.

53. Tantillo, M., Kesick, C. M., Hynd, G. W., y Dishman, R. K. (2001). The effects of exercise on children with attention-deficit hyperactivity disorder. Official Journal of the American College of Sports Medicine, 203-212.

54. Yamashita, Y., Mukasa, A., Anai, C., Honda, Y., Kunisaki, C., Koutaki, J., Tada, Y., Egami, C., Kodama, N., Nakashima, M., Nagamitsu, S. y Matsuishi, T. (2011). Summer treatment program for children with attention deficit hyperactivity disorder: Japanese experience in 5 years. Brain y Development (Official Journal of the Japanese Society of Child Neurology), 33, 260-267. 